Meta

Journal des traducteurs

Translators' Journal

\title{
Training Human Translators as Opposed to Programming Machine Translation Systems: A Performative Model
}

\section{Arvi Tavast}

Volume 50, numéro 4, décembre 2005

Pour une traductologie proactive - Actes

For a Proactive Translatology - Proceedings

Por una traductología proactiva - Actas

URI : https://id.erudit.org/iderudit/019894ar

DOI : https://doi.org/10.7202/019894ar

Aller au sommaire du numéro

Éditeur(s)

Les Presses de l'Université de Montréal

ISSN

0026-0452 (imprimé)

1492-1421 (numérique)

Découvrir la revue

Citer cet article

Tavast, A. (2005). Training Human Translators as Opposed to Programming Machine Translation Systems: A Performative Model. Meta, 50(4).

https://doi.org/10.7202/019894ar
Résumé de l'article

Dans cet article, je combine le modèle communicatif de traduction avec la linguistique performative pour en arriver à un modèle de traduction qui entend façonner pro-activement les attitudes des futurs traducteurs. L'idée autour de laquelle tourne ce modèle est que le traducteur, à l'instar de tout communicateur, a une intention communicative qui trouve son expression dans le texte cible. Il se situe ainsi à l'opposé de la traduction mécanique, dont l'objectif est de trouver des équivalents à des unités de traduction sans avoir en fait rien à dire dans la langue cible. Cet article se termine en indiquant une manière de construire pour les traductions un système d'évaluation fondé sur le modèle précédemment proposé. 


\title{
Training Human Translators as Opposed to Programming Machine Translation Systems: A Performative Model
}

\author{
ARVI TAVAST \\ Tallinn Pedagogical University, Tallinn, Estonia \\ arvi@imprimaatur.ee
}

\begin{abstract}
RÉSUMÉ
Dans cet article, je combine le modèle communicatif de traduction avec la linguistique performative pour en arriver à un modèle de traduction qui entend façonner pro-activement les attitudes des futurs traducteurs. L'idée autour de laquelle tourne ce modèle est que le traducteur, à l'instar de tout communicateur, a une intention communicative qui trouve son expression dans le texte cible. Il se situe ainsi à l'opposé de la traduction mécanique, dont l'objectif est de trouver des équivalents à des unités de traduction sans avoir en fait rien à dire dans la langue cible. Cet article se termine en indiquant une manière de construire pour les traductions un système d'évaluation fondé sur le modèle précédemment proposé.
\end{abstract}

\begin{abstract}
This paper combines the communicative model of translation with performative linguistics to arrive at a translation model that is meant to proactively shape the attitudes of future translators. Central to this model is the claim that the translator, like any communicator, has a communicative intent that gets expressed in the target text. This is contrasted with machine translation, which is concerned with finding equivalents to translation units without actually having anything to say in the target language. The paper concludes by indicating a way of building a translation evaluation system on the proposed model.
\end{abstract}

\section{MOTS-CLÉS/KEYWORDS}

translation process, translation quality, translator training, translation evaluation

\section{Introduction}

Based on various levels of equivalence between the source text and the target text, and limited to a couple of particular language combinations, translator training may have a striking similarity to programming a rule-based machine translation system. Attention is focused on bilingual dictionaries, contrastive grammar and parallel corpora: how to find, use and in some cases also create them. Translation is conceived as a linguistic, textual activity. The issue is: how is construct A in language $\mathrm{X}$ translated into language $\mathrm{Y}$ ?

It is a common sense notion that there can be some kind of direct relationship between (parts of) texts in different languages, and that this relationship might have something to do with translation. Unfortunately, this notion is still replicated in many translation training programmes. In a way, this is a successful educational strategy. Graduates indeed do produce target texts comparable to the state of the art in machine translation, "a type of literal translation which everyone condemns but which many practice" (Lederer 2003: 4).

This paper combines the communicative model of translation with performative linguistics (House 2001, Robinson 2003) and the protocol stack metaphor of network communications, to arrive at a translation model that is meant to proactively shape the attitudes of future translators. The intended change is away from deference to the dead letter of the source text, and towards the belief that the translator is a human communicator just like the original author. 


\section{Object of study}

The sole purpose of the proposed model is shaping the attitudes of students in translator training. Translator training in turn is mainly concerned with satisfying the needs of the mainstream translation market, which can be characterised as follows.

Globalisation has caused vendors to pay more attention to international markets. As these markets evolve, they increasingly require products to be localised, i.e. translated into their language(s) and adapted to local cultural needs. Market pressures have brought about the concept of simship - simultaneous shipping of a product in several languages, as opposed to shipping it in one language first and then localising into other languages. In a simship project timeline, translation is scheduled to overlap (if not coincide) with writing the original documentation. The next logical step is cooperative drafting - a process where several language versions are authored simultaneously by a team of writers.

In such settings, authors and translators are both anonymous and speaking for the initiator of translation, usually the vendor company. They have the same organisational status, being either employed or subcontracted by the initiator. They have similar competencies and responsibilities, with each author/translator being responsible for creating good quality documentation in each respective language. And finally, they are expected to work as a team, to help fulfil the overall business goals of the initiator, rather than pursue some separate agenda like the quest for equivalence in translation.

Prototypical examples of texts authored and translated in this way include user documentation, marketing material, packaging texts, software user interfaces, training material, web pages, news, articles in technical support knowledge bases, definitions in termbases, etc. In multilingual communities like Canada or the EU, this category also includes a large part of legislation. According to followers of Bühler's typology, these are exclusively informative and operative/vocative texts. Their translation is covert rather than overt (House 1982, 1997) and instrumental rather than documental (Nord 1997).

Expressly excluded from the object of this paper are most of the traditional main interests of translation studies:

- Translation of expressive texts (literary translation).

- Documental translation, i.e. situations where the initiator actually commissions a metatext about the original text rather than a target-language text about the original subject matter (translation of asylum applications when initiated by the authorities, of exam papers when initiated by the evaluator, etc.).

- Situations where the author is not anonymous and enjoys a higher status than the translator and the two are not working together in a team (translation of historic texts, of president's speeches when initiated by somebody else except the president's office, etc.).

- Descriptive translation studies.

- Tools for experienced translators.

The translation types in the first three items are excluded because of their relatively small market share both in terms of translation volume and especially in terms of turnover (considering that many of these types are usually under-paid). The latter two items are excluded due to different target groups: these two are of great interest for academic and professional audiences, while the model below is solely intended for translator training.

\section{The model}

In the ISO Open Systems Interconnection model of network communication (see e.g. http://www.iso.org/iso/en/CatalogueListPage.CatalogueList?ICS1=35\&ICS2=100), the encoding and decoding processes are subdivided into layers. When, for instance, intending to send an invoice to a trade partner, an accountant creates the invoice in the accounting program. This program instructs the message handling system to send a message to the intended recipient. Further down the layered protocol stack, the message is broken up into packets, frames and bytes, transformed into a continuous stream of ones and zeroes, and finally presented as a sequence of voltage changes 
in the cable. On the receiving side, everything is repeated in reverse order: ones and zeroes extracted from the signal in the cable, assembled into bytes, frames, packets and finally a message, then presented to the accounting program and ultimately received by the accountant of that company. The accountant will know whether the invoice should be ignored, disputed, paid immediately, paid only after receiving a reminder, escalated to a manager, etc.

Two points are worth noting here:

- The invoice is not transmitted. The only thing transmitted is the electrical signal in the cable.

- This signal on its own is completely meaningless. Moreover, intermediate stages in the (de)coding processes (packets, messages, even the invoice itself) are similarly meaningless. They acquire meaning only if we possess the right decoding mechanism, including enough accounting expertise to read an invoice and enough factual knowledge to understand what the invoice is for, and if we actually run the signal through this mechanism.

Just like in semiotics - meaning is not transmitted, it is constructed. A sign has no meaning unless a suitably qualified interpreter is interpreting it.

As a gross simplification, human communication can be described in a similar way. The electrical signal becomes ink marks on paper (or corresponding tokens in other media), and the protocol stack layers become linguistic levels from typography and orthography to pragmatics. Like above, everything that is transmitted, from ink marks to sentences to texts to semantically and even pragmatically analysed texts, is completely meaningless in the absence of an interpreter. In ordinary monolingual communication, the hearer is the interpreter who completes the process and makes communication work.

Introducing a second language into this model brings along some kind of mediator between the languages. Here we have another handy analogy with network communication: devices that interconnect different (parts of) networks. A repeater for instance is a device that understands ones and zeroes, is able to forward them from one type of cable to another, but does not understand anything else. The counterpart in human communication might be a typist: able to literally transcribe a text, but not paying any attention to words or anything above that. A router additionally understands packets and can translate them into packets of another network. The counterpart might be a translator who uses a bilingual dictionary for producing a word-for-word translation. On the next level of sophistication, gateways might be seen as corresponding to the translation of sentences or paragraphs as illustrated on figure 1. This is a widespread variety, represented by a large number of ordinary poor-quality human translators and, interestingly for the topic of this paper, the state of the art in machine translation. 
Figure 1. Machine translation as performed by machines or poor-quality human translators.

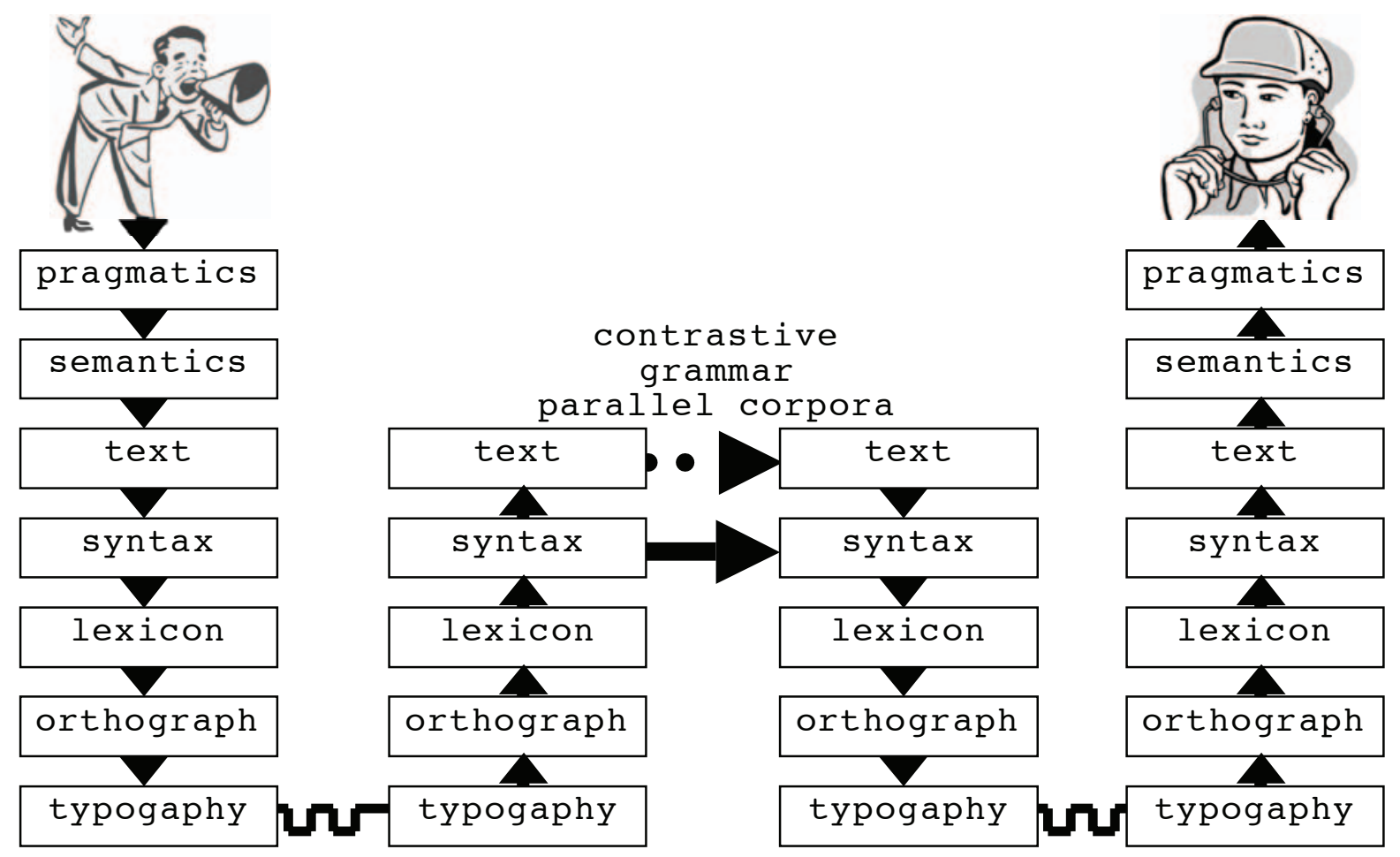

It is possible to go further, including also semantic and pragmatic analysis and synthesis and finding functional or dynamic equivalents to whole texts. After that, the model starts looking very similar to Roger Bell's model of the translation process (Bell 1991). This model is applied both to programming machine translation systems and to training human translators in schools concerned with how construct $\mathrm{A}$ in language $\mathrm{X}$ is translated into language $\mathrm{Y}$. The issue on the pragmatics level is how to transfer the communicative intent of the original author into the target language (e.g.

Hervey 1998, House 1998). This does not differ fundamentally from transfer rules on lower levels. Teaching is still an exercise of programming essentially dumb machines to follow rules, only the rules have been made more elaborate.

From such a model that contains analysis and synthesis on all linguistic levels, there is still one element missing: the human translator. This is where performative linguistics comes into play (House 2001, Robinson 2003). Good-quality human translators have their own communicative intent that they express in the target text, they do things with words in their translation, rather than trying to convey what original authors have done with words in the source text. 
Figure 2. Human translation, including the translator's own communicative intent.

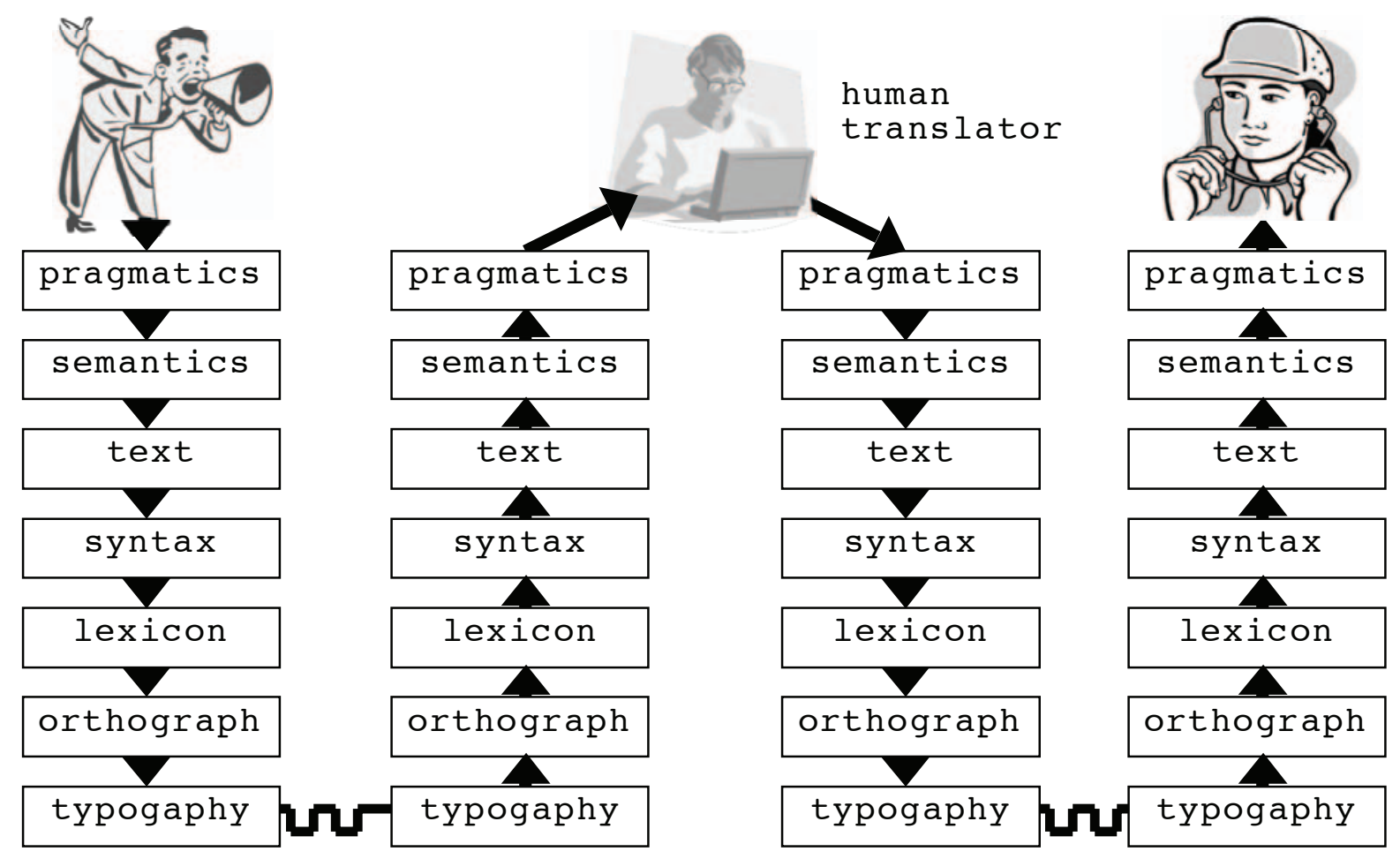

It should be stressed that the proposed model does not attempt to describe or explain how communicative intent works; it is taken as a property of the human black box. Of course, sooner or later will cognitive sciences succeed in describing, explaining and modelling the human mind, as well as imitating it in software, which will make obsolete not only the opposition in the title of this article but also translator training as such. Until that happens, however, we can continue opposing quality translation to translation done using transfer rules on whatever linguistic level without the participation of a human mind.

\section{Discussion}

Practicing translators sometimes talk about the anecdotal fear of machines taking over. Will I lose my job as a result of developments in machine translation? The answer, unfortunately, is yes. If you translate like a machine, then a real machine can do the same thing faster, cheaper and more reliably. The only thing that can be done to compete with machines in the medium term is to provide services that the machines are not yet capable of providing.

No machine is currently able to have a communicative intent (or want anything else for that matter). With advances in technology, it is increasingly possible to make a machine emulate this intent and behave in pre-programmed situations as if it was there. It is even more possible to train human translators procedurally to conceal their absence of communicative intent. The problem is that the result is not universal - it only works in situations that have been foreseen by the instructor (programmer). As soon as something unexpected happens, for instance when no match is found in dictionaries or parallel corpora or when these contain information that is not true, the system breaks down and starts producing unpredictable output.

Another revealing analogy is teaching a person to walk. In theory, this could be done by describing which muscles to contract or relax in which order, and telling the learner how to base "walking decisions" on various types of tactile feedback and information from the balance organ. In practice, such a description would be either too complicated to follow or too much simplified to 
really work. A similarity can indeed be noticed between the failure to build a two-legged walking robot and the failure to achieve fully automatic high quality translation. Both are trivially easy for many humans, but prohibitively difficult to program (or teach) in a procedural way.

The traditional objection to human translation in the sense described above is that translators are not free in their creative decisions. "Unlike wholly creative writing, that is to say the production of an original text, translating is morally neutral, the translator's job being to translate what is there." (Weston 2003: 149, original emphasis.) But there's nothing there. It would be very difficult to prove that the ink marks on the paper where the original text is written objectively mean something without the help of an interpreter.

Also, if such constraints are placed on the translator, why not on the original writer? Why should the documentation of a product be written by a creative human in one language and mechanical text-transcoders in all others? Why is this one language so special? The simple answer is: it is not. International readers expect good user manuals in their language, not linguistic studies about the original manual. This is well known to experienced translation customers. Their criteria for translation evaluation often require that the translation should adequately describe the product, with nothing being said about any kind of correspondence with the source text. The source text is simply not relevant. It is just another language version of the document, possibly containing errors, possibly written in a poor style, etc.

Even a perfect machine translation system, a system that is capable of fully analysing and synthesizing all linguistic levels, differs fundamentally from a human communicator in that it fails to understand the point that the original author was trying to make. Without understanding, however, there is no way it could make sense of novel language uses, expression choices of doubtful suitability or outright errors made by the author. The human communicator has no difficulty with these. Neither has a human translator.

This is why the attitude towards correction of source text errors is a good indicator of whether the translator behaves like a human or like a machine. Why would a responsible human communicator knowingly say something that is not true? Only if actually commissioned to do that in a non-cooperative communication situation like advertising or legal discourse. But if the original author has inadvertently made a mistake in the original, i.e. has meant one thing and written another, then the only possible course of action for a human translator is to find a way to correct it.

How does the translator know what the author really meant? Explaining in detail how people communicate is neither the objective of this paper nor among the competencies of its author. It is sufficient to observe that in a vast majority of cases, communication between cooperation-oriented humans works at least satisfactorily. Almost always does the hearer obtain some kind of understanding not only about what was said, but also why it was said, what kind of impression did the speaker want to leave or what the speaker really meant. This works in the same way in translation. Provided sufficient communicative competence, the translator acts as a normal hearer in the source language.

Here the traditional objection is that translators can not afford acquiring such a high level of communicative competence in all subject fields that they translate. If this is the case, then there are three options: specialise narrower, continue in direct competition with machine translation, or find a less demanding job. Quality translation without expert-level communicative competence in both languages is not possible.

\section{Evaluation system}

The use of this model in translator training can be enforced by also using it for providing formative feedback. If the translation process is conceived in a simplified way as proceeding linearly through this model, then it is possible to locate on the model the source of each error.

There are two large groups:

- Decoding errors or "short circuits". These happen when source text analysis fails at a certain linguistic level and the translator jumps directly to synthesis of the same level. A frequent example is the lexical short circuit, i.e. uncritical use of a bilingual dictionary. 
- Coding errors. These are exactly the same as potential errors in normal monolingual writing. Examples include anything from violation of politeness conventions to double spaces between words.

Each of these types can be subdivided according to layers of the model. If a marking system such as Markin (http://www.cict.co.uk/software/markin/index.htm) is used, each layer can in turn be populated with a set of recurring error types complete with standard explanatory texts. Then all the evaluator has to do most of the time is assign an error type to each issue found in a student translation. Only rare or peculiar errors need a personal comment.

If summative evaluation is needed, each of these error categories can be assigned a weight and a weighed total can be calculated. To support the attitude change suggested here, decoding errors should have much higher weights than coding errors. While no evaluation method is entirely free of subjectivity, this total at least ensures consistent grading across students within a particular assignment.

This evaluation system has been used in translation classes at Tallinn University since 2003 and it has kept both instructors and students happy. The former have got a noticeable increase in marking speed and the latter have received more individual feedback and an impression of relatively objective grading.

\section{Conclusion and future directions}

Surely there will be a time when comparison to low-quality human translators will be an insult for machine translation systems. But this can only happen with systems capable of feeling insulted, and that's still some time away. Until then, this comparison can be used in translation classes for illustrating the difference between good and bad translation.

Without attempting to explain the inner workings of the human mind, it has been claimed in this paper that for quality translation, a human mind is unavoidable. The only property that distinguishes a human from machine translation systems of today or the near future, is the ability to have a communicative intent as well as to understand the intent of the original author. If students are taught deference to the source text and told that they are not allowed to have their own communicative intent, then they face direct competition from machine translation systems. The solution is to do the opposite - use every opportunity to encourage students to forget the source text and behave like responsible human communicators. This can be supported by an evaluation system that stresses the results of any fallback into mechanical transcoding.

The next step is to gather empirical evidence about the existence of a correlation between attitudes and translation quality, both across subjects and within the same subject over time. That will show whether attitudes are worth influencing. Also, it would be interesting to see whether attempts to influence attitudes really work, or which approaches work better.

\section{REFERENCES}

BELL, R. (1991): Translation and Translating, London/New York, Longman.

HERVEY, S. G.J. (1998): "Speech Acts and Illocutionary Function in Translation Methodology", in HiCKEY L. (ed.): The Pragmatics of Translation, Clevedon/Buffalo/Toronto/Sydney, Multilingual Matters Ltd, p. 10-24.

House, J. (1982): A Model for Translation Quality Assessment, 2nd edition (1st edition 1977), Tübingen, Gunter Narr Verlag.

House, J. (1997): Translation Quality Assessment: A Model Revisited, Tübingen, Gunter Narr Verlag.

House, J. (1998): "Politeness and Translation", in HickeY L. (ed.) The Pragmatics of Translation,

Clevedon/Buffalo/Toronto/Sydney, Multilingual Matters Ltd, p. 54-71.

House, J. (2001): "Pragmatics and Translation", in Pathways of Translation Studies, Centro Buendía, Universidad de Valladolid, p. 57-78.

LEDERER, M. (2003): Translation. The interpretive model. Translated by Ninon Larché, Manchester/Northampton, St. Jerome Publishing. 
NoRD, C. (1997): Translating as a Purposeful Activity. Functionalist Approaches Explained, Manchester/Northampton, St Jerome Publishing.

Robinson, D. (2003): Performative Linguistics: Speaking And Translating As Doing Things With Words, New York/London, Routledge.

Weston, M. (2003): "Meaning, Truth and Morality in Translation”, in Anderman, G. and Rogers M. (eds.): Translation Today: Trends and Perspectives, Clevedon/Buffalo/Toronto/Sydney, Multilingual Matters Ltd, p. 140151. 